\title{
Estimation of the contribution of gaps to tritium retention in the divertor of ITER
}

\author{
D. Matveev ${ }^{1,2}$, A. Kirschner ${ }^{1}$, K. Schmid ${ }^{4}$, A. Litnovsky ${ }^{1}$, D. Borodin ${ }^{1}$, M. Komm ${ }^{3}$, \\ G. Van Oost ${ }^{2}$ and U. Samm ${ }^{1}$ \\ ${ }^{1}$ Institut für Energie- und Klimaforschung - Plasmaphysik (IEK-4), \\ Forschungszentrum Jülich GmbH, Association EURATOM-FZJ, Trilateral Euregio \\ Cluster, Wilhelm-Johnen-Straße, D-52425 Jülich, Germany \\ ${ }^{2}$ Department of Applied Physics, Ghent University, Sint-Pietersnieuwstraat 41 B4, \\ 9000 Gent, Belgium \\ ${ }^{3}$ Institute of Plasma Physics AS CR, v.v.i., Za Slovankou 3, 18200 Prague 8, Prague, \\ Czech Republic \\ ${ }^{4}$ Max-Planck-Institut für Plasmaphysik, Boltzmannstraße 2, D-85748 Garching b., \\ München, Germany \\ E-mail: d.matveev@fz-juelich.de
}

\begin{abstract}
An estimation of the contribution of gaps to beryllium deposition and resulting tritium retention in the divertor of ITER is presented. Deposition of beryllium layers in gaps of the full tungsten divertor is simulated with the 3D-GAPS code. For gaps aligned along the poloidal direction, non-shaped and shaped solutions are compared. Plasma and impurity ion fluxes from [K. Schmid, Nucl. Fusion 48 (2008) 105004] are used as input. Ion penetration into gaps is considered to be geometrical along magnetic field lines. The effect of realistic ion penetration into gaps is discussed. In total, gaps in the divertor are estimated to contribute about $0.3 \mathrm{mgT} / \mathrm{s}$ to the overall tritium retention dominated by toroidal gaps, which are not shaped. This amount corresponds to about 7800 ITER discharges up to the safety limit of $1 \mathrm{~kg}$ in-vessel tritium; excluding, however, tritium release during wall baking and retention at plasma-wetted and remote areas.
\end{abstract}

PACS: 52.40.Hf; 52.25.Vy; 52.65.Pp

\section{Introduction}

Erosion-deposition behaviour of plasma-facing materials and especially the aspects of material life time and tritium retention are the most critical for the availability of future fusion devices such as ITER. Material erosion, migration, re- and co-deposition processes have the strongest impact on the in-vessel retention of radioactive tritium $(\mathrm{T})$, which must be limited $(\sim 1 \mathrm{~kg})$ and controlled for safety reasons. Remote plasma-shadowed areas in the divertor and gaps in castellated plasma-facing components (PFCs) are the potential sites for co-deposition of fuel with plasma-facing materials eroded elsewhere in the device. The ITER divertor will consist of around 320,000 individual monoblocks with a spacing of $\sim 1 \mathrm{~mm}$ and a gap depth reaching $\sim 30 \mathrm{~mm}$ [1]. Effective removal of tritium retained in co-deposits in such gaps represents a challenging task. In ITER, co-deposition of T will happen substantially with beryllium (Be) eroded from the first wall, therefore modelling concerning Be transport and re-deposition in ITER, especially inside gaps of castellated PFCs, is essential to study the possible influence on the ITER duty cycle. Modelling of Be transport and redeposition at plasma-wetted areas in the divertor of ITER has been performed earlier [2-4]. This paper presents an estimation of the contribution of gaps in a full tungsten (W) divertor of ITER to beryllium deposition and resulting tritium retention by co-deposition.

\section{Model assumptions}


Be deposition in gaps in the divertor of ITER will be governed by the total influx of Be into the divertor area, its redistribution over the targets, baffles and dome surfaces, local plasma penetration into gaps and the overall re-deposition efficiency inside gaps, which will also depend on the gap geometry. In general, the deposition rate of $\mathrm{Be}$ in an individual gap can be written as follows:

$$
d N_{B e^{g a p}} / d t=\eta \times \alpha \times \Gamma_{B e} \times A,
$$

where $\Gamma_{B e}$ provides the flux of beryllium to the PFCs surface at the location of the gap, $A$ corresponds to the PFCs area occupied by the gap aperture, $\alpha$ gives the fraction of the Be flux that eventually enters the gap aperture, and $\eta$ represents the probability for the incoming Be particles to be trapped inside the gap (hereafter referenced as the gap trapping probability). All parameters of equation (1) depend on the actual gap position within the divertor. The total deposition rate of $\mathrm{Be}$ in gaps can be calculated by integration over the entire divertor surface, which can be reduced to integration over the poloidal distance $s$ if toroidal symmetry is assumed:

$$
d N_{B e} / d t=\delta_{A} \int_{s} \eta(s) \times \alpha(s) \times \Gamma_{B e}(s) \times 2 \pi R(s) \times d s
$$

where the gap trapping probability $\eta$, the Be flux $\Gamma_{B e}$ and the major radius $R$ at gap locations are functions of the poloidal coordinate $s$. In general, due to the variation of the gap width and monoblock dimensions with the poloidal location, the area fraction $\delta_{A}$ occupied by gaps is also a function of $s$. However, it is not straightforward to account for such a variation. To simplify the integration, it is assumed that $\delta_{A}$ is constant over the entire area of the divertor. For a representative monoblock $30 \mathrm{~mm}$ in the toroidal direction and $12 \mathrm{~mm}$ in the poloidal direction and a gap width between monoblocks of $1 \mathrm{~mm}$ both toroidally and poloidally, $\delta_{A-p o l} \approx 3.2 \%$ for gaps aligned along the poloidal direction (poloidal gaps) and $\delta_{A-t o r} \approx 7.7 \%$ for gaps aligned along the toroidal direction (toroidal gaps).

To evaluate equation (2), the fluxes of Be ions and atoms in the divertor were taken from [2]. In that work, calculations of $\mathrm{Be}$ erosion at the main wall were combined with plasma transport and erosion/deposition simulations to account for re-erosion and re-deposition of $\mathrm{Be}$ in the full $\mathrm{W}$ divertor of ITER. Figure 1 shows the poloidal profile of the ITER divertor and its discretization as used in the Be flux distribution calculations [2], which was also adopted in the present work for the calculations of the gap trapping probability $\eta$. The deuterium (D) plasma background with argon (Ar) as the main radiating impurity from B2/EIRENE calculations [4] was used. The choice of this plasma background was motivated by the intention to exclude carbon (C) from consideration, thus no chemical erosion and no formation of ternary alloys in the modelling. The $\mathrm{D}$ plasma was used since there was no $\mathrm{D} / \mathrm{T}$ plasma background without $\mathrm{C}$ available for ITER. For consistency, the same plasma background was also used in the present work. Except for Ar seeding, this plasma background corresponds to a high power ITER reference discharge with $130 \mathrm{MW}$ power going into the SOL. The poloidal profiles of the $\mathrm{D}^{+}$ion flux, mean impact energy, and the Be flux fractions used in the present work are shown in figures 7 and 11 in [2]. For the integration of equation (2) the profiles of Be flux fractions were interpolated between given data points independently for all charge states.

To calculate the Be gap trapping probability $\eta$, the 3D-GAPS code [5] was applied. Deposition of Be inside poloidal gaps was modelled using the TRIM code data for Be reflection and accounting for physical sputtering of $\mathrm{Be}$ due to $\mathrm{D}$ ions and self-sputtering. The sputtering yields used in the present work represent the so-called "ERO-min" estimate $[6,7]$ corresponding to the assumption of $50 \% \mathrm{D}$ concentration in the surface layer and are calculated based on the fit formulae for the sputtering yield from [8]. Possible chemical erosion of Be by D [9] was neglected since its contribution under tokamak conditions is rather uncertain. The information on plasma penetration into gaps represents an important input for 3D-GAPS simulations. Typically, such information is obtained from Particle-In- 
Cell (PIC) simulations, e.g. with the SPICE2 code [10]. However, due to limitations that will be described in section 3.3, 3D-GAPS simulations of poloidal gaps presented in this work were performed assuming a simple geometrical, i.e. along magnetic field lines, ion penetration into gaps, which effectively corresponds to parameter $\alpha=1$ in equation 2 . For simplicity, it was assumed that magnetic field lines hit the divertor surfaces at a constant angle of $3^{\circ}$ throughout the entire divertor. For the gap width of $1 \mathrm{~mm}$, this corresponds to a plasma-wetted area of $0.05 \mathrm{~mm}$ in a poloidal gap (leading edge). From experiments [11] and modelling [12] it is known that toroidal gaps also can receive even larger fluxes of incoming particles and therefore may show large impurity re-deposition that is at least comparable to the poloidal gaps. Ion penetration into toroidal gaps can not be evaluated from simple geometrical considerations; therefore in this work toroidal gaps were not simulated. Instead, an assumption was made that toroidal gaps show the same ion penetration and the same redeposition efficiency as the poloidal gaps. Unlike ions, neutral Be atoms were assumed to enter the gaps isotropically.

From the resulting Be deposition rates, an estimation of the $\mathrm{T}$ retention in deposited Be layers inside gaps was made based on the assumption of a constant $\mathrm{T} / \mathrm{Be}$ ratio of 0.05 in the deposits [13], same as was used in [3], i.e. $(\mathrm{T}+\mathrm{D}) / \mathrm{Be}=0.1$.

\section{Results and discussion}

\subsection{Beryllium deposition and tritium retention}

The poloidal profile of the total Be influx into the gaps based on the data from [2] is shown in figure 2 . As a result of a set of 3D-GAPS simulations performed for poloidal gaps in various regions of the divertor with the corresponding variation of plasma parameters and charge-resolved Be fluxes impacting on PFCs, the poloidal dependence of parameter $\eta$ in equation (2), i.e. the gap trapping probability, was reconstructed (figure 2). Variation of $\eta$ across the divertor is due to different incoming Be flux fractions and impact energies of ions, which together affect reflection and sputtering of Be and thus the erosion/deposition balance at plasma-wetted areas and in the gaps. The gap trapping probability varies in the range from 0.2 to 0.6 except for locations near the strike points and in the private flux region where $\eta$ tends to unity due to extremely low plasma temperatures of about $1 \mathrm{eV}$, which lead to zero or extremely low Be sputtering yields and almost zero Be reflection (from deposited Be layers). At locations where simultaneously a high $\mathrm{D}$ flux and a high plasma temperature are observed, the gap trapping probability decreases due to re-erosion of deposited Be layers. The smallest values of $\eta$ correspond to locations where plasma-wetted areas inside gaps are erosiondominated and thus show large reflection probabilities of $\mathrm{Be}$ on $\mathrm{W}$.

Accounting for the Be flux distribution along the divertor targets, the gap trapping probability $\eta$ and the total toroidal length of the divertor at the radial positions $R$ corresponding to each given poloidal location, equation (2) can be integrated to get the total Be deposition rate in the gaps in the divertor. As a result, the rates of $3.7 \times 10^{20} \mathrm{~s}^{-1}$ for all poloidal gaps and $8.9 \times 10^{20} \mathrm{~s}^{-1}$ for all toroidal gaps were obtained. Under the assumption of the average $\mathrm{T} / \mathrm{Be}=0.05$ in deposited layers, the amount of tritium retention in gaps in the divertor is estimated to be about $0.09 \mathrm{mgT} / \mathrm{s}$ for poloidal gaps and $0.22 \mathrm{mgT} / \mathrm{s}$ for toroidal gaps. It has to be noted that the difference between poloidal and toroidal gaps seen in the present work is only due to different total areas occupied by gaps. In total gaps in the divertor of ITER could contribute up to about $0.3 \mathrm{mgT} / \mathrm{s}$ to the overall tritium retention. This amount, attributed mostly to toroidal gaps, would correspond to $\sim 0.13 \mathrm{~g}$ of tritium accumulated per one $400 \mathrm{~s}$ long ITER discharge or about 7800 ITER discharges before the safety limit of $1 \mathrm{~kg}$ (retention at plasma-wetted and remote areas excluded). It has to be noted that although simulations correspond to an ITER discharge with Ar seeding, sputtering of Be layers in gaps by Ar impurity was not taken into account in 3D-GAPS modelling.

\subsection{Gaps versus plasma-wetted areas}


Simulations with the ERO code have been performed earlier to predict the amount of tritium retention at plasma-wetted surfaces. The results presented in [3] were obtained for CFC targets assuming the constant Be flux fraction of $0.1 \%$ for the outer divertor and $1 \%$ for the inner divertor (no variation along the divertor targets). The retention rate of $0.3 \mathrm{mgT} / \mathrm{s}$ obtained in the previous section would correspond to the gap contribution of about $4 \%$ to the overall tritium retention if compared to about $7 \mathrm{mgT} / \mathrm{s}$ retention in Be layers predicted for plasma-wetted areas on the targets [3] using the same assumption of $(\mathrm{D}+\mathrm{T}) / \mathrm{Be}=0.1$. However, one has to keep in mind that although the Be flux profile used in the present work corresponds to larger average Be fractions $(\sim 0.6 \%$ outer divertor and $\sim 1.7 \%$ inner divertor), the overall $\mathrm{Be}$ influx into the divertor area is about 70 times smaller [14].

\subsection{Effect of realistic ion penetration into gaps}

Due to the space discretization into cells of the size of the Debye length $\lambda_{D}$, the computational time of PIC simulations increases with decreasing $\lambda_{D}$. This restricts the range of plasma parameters that can be simulated by PIC for a castellation of a given size. In case of the SPICE2 code, a reasonable computational time for the ITER castellation can be achieved only for plasma parameters corresponding to locations about $40 \mathrm{~cm}$ away form the strike points (normal operation, the entire monoblock of a $30 \mathrm{~mm}$ toroidal length included in the simulation domain). Simulations for such locations with $\mathrm{n}_{\mathrm{e}}=2 \times 10^{19} \mathrm{~m}^{-3}$ and $\mathrm{T}_{\mathrm{e}}=12 \mathrm{eV}$ were performed that indicate that plasma flux entering the gaps is a factor 4 reduced compared to simple geometrical assumptions (figure 3 ). This behaviour is due to ion deflection by the positive electrostatic potential hump formed right at the gap entrance. Locations closer to the strike points could not be simulated, however the ion transport in gaps at higher densities and lower temperatures can be expected to be even more reduced [10].

\subsection{Effect of gap shaping}

Toroidal chamfering of monoblocks is considered as one of the measures to protect leading edges in the high heat flux area of the ITER divertor [15]. Partial chamfering of monoblocks from one side and rounding of the corner on the other side is investigated experimentally in TEXTOR and other machines [16] as an optimized solution for ITER. Predictive modelling performed with the 3D-GAPS code suggests that chamfering of monoblocks would decrease impurity deposition due to ions manifold. However, the shaped gaps still remain open for incoming neutrals. As for the plasma penetration, it has to be noted that 3D-GAPS simulations were performed assuming geometrical shadowing of neighbouring monoblocks. It was demonstrated earlier with SPICE2 simulations that plasma can penetrate even into shaped gaps [10]. SPICE2 simulations of gaps shaped as in [16] indicate that ions cannot penetrate into the gap itself (i.e. below the corner of the chamfered side). However, the rounding on the left side of the gap still receives a significant ion flux that is only about a factor 6.5 smaller than that shown by SPICE2 at the leading edge of the non-shaped gap. Accounting for the ion fluxes from SPICE2 and assuming the same effect on the transport of Be ions, the resulting Be deposition in shaped gaps is at least a factor 10 reduced compared to non-shaped gaps if neutral sources are excluded. Considering simple geometrical ion transport along the magnetic field lines and accounting for the contribution of neutral Be, gap shaping results in a factor 2 to several hundreds reduction of the deposition at different poloidal locations in the divertor, depending on the neutral $\mathrm{Be}$ flux fraction. For few locations at the dome with very high neutral Be flux fractions, namely locations in front of the strike points, no mitigation of deposition at all is observed. Ultimately, the poloidal gap shaping alone is not sufficient to decrease the tritium retention in gaps significantly due to dominating fuel accumulation in toroidal gaps.

\section{Summary}

Modelling of Be transport and re-deposition in gaps of castellated surfaces in the divertor of ITER was performed using the 3D-GAPS code. An estimation of tritium retention in deposited Be layers in gaps 
was provided. Assuming geometrical plasma penetration into gaps, the total retention rate of about $0.3 \mathrm{mgT} / \mathrm{s}$ was predicted for conventional (non-shaped) castellation blocks. This rate is dominated by toroidal gaps and corresponds to about 7800 ITER discharges before the safety limit of $1 \mathrm{~kg}$ tritium. The retention in gaps is estimated to contribute only about $4 \%$ to the overall $\mathrm{T}$ retention in the divertor. According to PIC simulations, realistic plasma penetration into gaps must be smaller than estimated from the simple geometrical assumption used in the present work. The ion flux reduction is due to formation of electrostatic potential hump below the gap entrance. This effect will most probably reduce the retention, however quantification is not straight-forward. In addition, experiments [17] indicate that the long-term isothermal annealing at a temperature of $623 \mathrm{~K}\left(350{ }^{\circ} \mathrm{C}\right)$ can reduce the amount of retained fuel by $20-50 \%$ depending on the $\mathrm{W}$ admixture into Be layers deposited at temperatures lower than the baking temperature. However, for layers grown at comparable or higher temperatures annealing does not lead to efficient fuel removal. Shaping of poloidal gaps is predicted to be beneficial for the mitigation of impurity deposition in the gaps; however it is less efficient for locations with high neutral fluxes and has only minor effect against the major deposition occurring in non-shaped toroidal gaps.

\section{Acknowledgements}

The work presented here was supported by the Research Fund of Ghent University (BOF), Belgium.

\section{References}

[1] R. Pitts et al., J. Nucl. Mater. 415 (2011) S957-S964

[2] K. Schmid, Nucl. Fusion 48 (2008) 105004

[3] A. Kirschner et al., J. Nucl. Mater. 390-391 (2009) 152-155

[4] A.S. Kukushkin and H.D. Pacher, Plasma Phys. Control. Fusion 44 (2002) 931-943

[5] D. Matveev et al., Plasma Phys. Control. Fusion 52 (2010) 075007

[6] D. Borodin et al., J. Nucl. Mater. 438 (2013) S267-S271

[7] D. Borodin et al., these proceedings

[8] W. Eckstein and R. Preuss, J. Nucl. Mater. 320 (2003) 209-213

[9] C. Björkas et al., J. Nucl. Mater 438 (2013) S276-S279

[10] M. Komm et al., Plasma Phys. Control. Fusion 53 (2011) 115004

[11] A. Litnovsky et al., J. Nucl. Mater. 390-391 (2009) 556-559

[12] R. Dejarnac et al., J. Nucl. Mater. 415 (2011) S977-S980

[13] M. Baldwin et al., J. Nucl. Mater. 337-339 (2005) 590-594

[14] A. Kirschner et al., Phys. Scripta T138 (2009) 014011

[15] S. Carpentier et al., these proceedings

[16] M. Hellwig et al., these proceedings

[17] K. Sugiyama et al., J. Nucl. Mater. 438 (2013) S1113-S1116 


\section{Figure captions}

Figure 1. The poloidal profile of the ITER divertor and its discretization (wall element indexes) as used in the Be flux distribution calculations in [2].

Figure 2. Poloidal profiles of the total Be influx into the gap based on the data from [2], and the gap trapping probability $\eta$ obtained by the 3D-GAPS modelling. Circles correspond to simulated locations. The solid line represents the fitted curve. Counting of the poloidal coordinate starts at the upper part of the outer baffle at $(\mathrm{R}, \mathrm{Z})=(6.3302,-3.0358)$ and goes clockwise up to the upper part of the inner baffle at $(R, Z)=(4.3375,-2.6302)$.

Figure 3. Ion flux along the plasma-open side inside a non-shaped poloidal gap resulting from the SPICE2 simulations for the plasma parameters above the gap $n_{e}=2 \times 10^{19} \mathrm{~m}^{-3}$ and $\mathrm{T}_{\mathrm{e}}=12 \mathrm{eV}$. The grey area corresponds to the geometrical plasma-wetted area (along the magnetic field lines). The dashed line shows the ion flux at the top surface of the monoblock. 


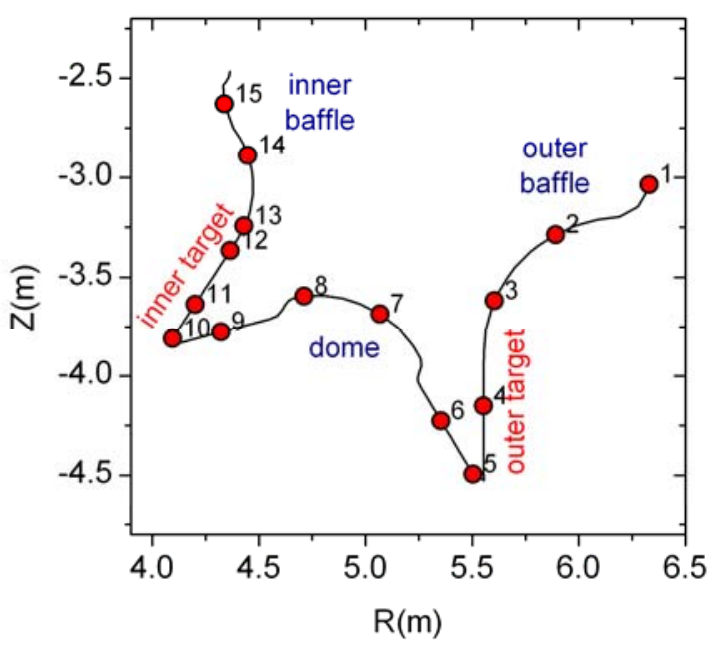

Figure 1. The poloidal profile of the ITER divertor and its discretization (wall element indexes) as used in the Be flux distribution calculations in [2]. 


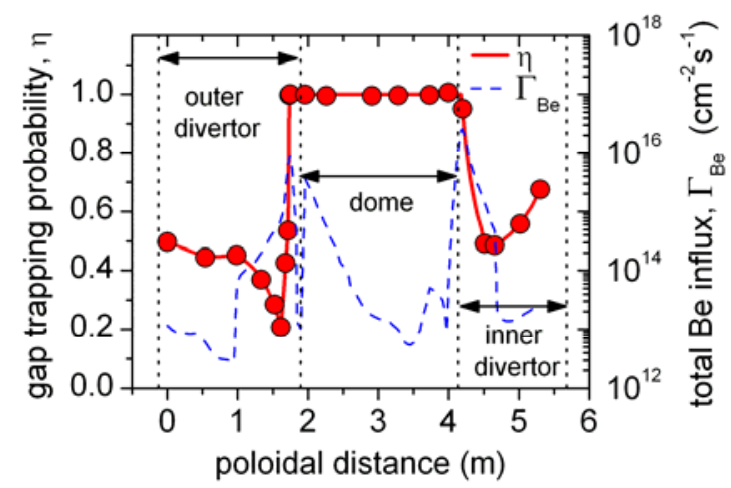

Figure 2. Poloidal profiles of the total Be influx into the gap based on the data from [2], and the gap trapping probability $\eta$ obtained by the 3D-GAPS modelling. Points correspond to simulated locations. The solid line represents the fitted curve. Counting of the poloidal coordinate starts at the upper part of the outer baffle at $(\mathrm{R}, \mathrm{Z})=(6.3302,-3.0358)$ and goes clockwise up to the upper part of the inner baffle at $(\mathrm{R}, \mathrm{Z})=(4.3375,-2.6302)$. 


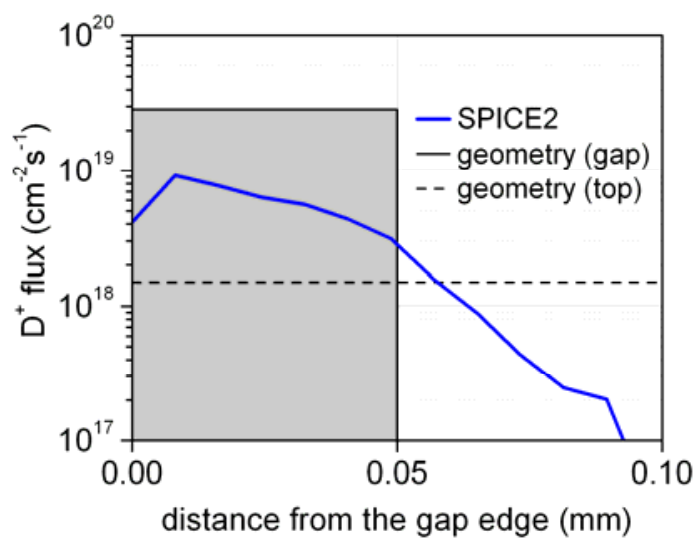

Figure 3. Ion flux along the plasma-open side inside a non-shaped poloidal gap resulting from the SPICE2 simulations for the plasma parameters above the gap $\mathrm{n}_{\mathrm{e}}=2 \times 10^{19} \mathrm{~m}^{-3}$ and $\mathrm{T}_{\mathrm{e}}=12 \mathrm{eV}$. The grey area corresponds to the geometrical plasma-wetted area (along the magnetic field lines). The dashed line shows the ion flux at the top surface of the monoblock. 